\title{
Nanoparticle Based Water Treatment Model in Squeezing Channel under Magnetic Field
}

\author{
Sergei Zuev ${ }^{1, *}$, Zakir Hussain ${ }^{2}$ and Petr Kabalyants ${ }^{1}$ \\ 1 Belgorod State Technological University named after V.G. Shukhov, Belgorod, Russia; sergey.zuev@bk.ru \\ 2 COMSATS University Islamabad, Abbottabad Campus, Abbottabad, Pakistan; zakirali@ciuatd.edu.pk \\ * Correspondence: sergey.zuev@bk.ru; Tel.: +7 4722549853
}

\begin{abstract}
The process of water treatment by nanoparticles is one of the most considerable subjects in the cross-field of hydrodynamics, chemistry and mathematics. This paper is dedicated to the case of the flows that appear when squeezing and stretching a spongy with a mix of water with nanoparticles and contaminants. It is assumed that fluid is homogeneous at the starting moment, the parameters of the nanoparticles and contaminants are known, and there is a constant non-homogeneous magnetic field applied to the system. The flow starts moving when the walls of the channel shift to each other. Exact and numerical solutions of the system of ordinary differential equations are used to receive the results. The article gives an answer to the question about stability of the flow and proposes the technique to evaluate the essential characteristics of the system to achieve the treatment process efficiency. The main result is that the considered system shows excellent treatment properties during some part of squeezing stage. This effect does not appear without magnetic field.
\end{abstract}

Keywords: exact solutions, instability of the flow; nano-fluid; nanoparticles removing; magnetohydrodynamics; stability of the flow; water treatment.

\section{Introduction}

Magneto-Hydro-Dynamics (MHD) is the crossroad of physical and mathematical methods which are used to analyze the motion of fluids in magnetic or electro-magnetic field. The triple word magneto-hydro-dynamics consists of "magneto" means magnetic, "hydro" means liquid and "dynamics" refers to the object movement due to forces. Sometimes MHD is named as "hydro-magnetics" or "magneto-fluid-dynamics".

Hannes Alfven [1], a famous Swedish research analyst first explored the MHD fluid. His contribution in the field of plasma physics is remarkable. The magnetic field proves an electric current in the flowing conducting fluid. The electric current flowing through the conductive fluid generates a force on the fluid and affects the magnetic field. The electrically conductive flows were analyzed by numerical simulation in various applications, such as, in parallel film [2-3], applied magnetic field [4], and treating of polymer in microgravity [5].

There are two problems among the whole specter of MHD interests that concern the present paper subject. The first one is common enough and it is called stability problem. It is targeted to answer the question "what are the conditions for the flow to become instable?" Of course, the magnetic field influence is taken into account first of all. The second problem is particles association both for nanoparticles and for contaminants. It attempts to answer the question "how magnetic field and flow velocity impact on the concentrations scalar fields?"

The latest references concerning instability explorations can be illustrated by [2-5]. Stability of two fluids MHD flow inside a flat channel was explored by Z. Hussain et al. in [5] where the corresponding exact solution has been presented. Of course, there are a lot of papers and conferences were devoted to this problem, but we just have to appoint 
the work [6] of A.S. Dalkılıç et al. where the heat conduction of hybrid nanofluid CNT$\mathrm{SiO}_{2}$ with water was analyzed experimentally; these results are close to those in the present paper, in spite of the difference in physical background. Numerical simulations of the MHD flow stability in rectangular channel were made in [7]. Stability and some other physical aspects have been discussed in [8] with respect of the problem of water treatment.

The second problem appointed above is under consideration in the paper [9] where polymerization problem has been explored. The concentrations are closely related to heat transfer. That is why most methods that have been used for heat transfer explorations are applicable to concentrations analysis as well. Thus, Jing et al. [10] studied the heat transfer with the help of five distinct sorts of nanoparticles in unsteady flow of nanofluids.

The results of a magnetic field influence on the flow of heat transmission of $\mathrm{Fe}_{2} \mathrm{O}_{3}$ with a uniform heat flux was examined by Kandelousi [11]. He examined flow of nanofluids and heat transmission across the absorbent medium.

Nanofluids have many applications in different industries such as in automotive industry it is used for enhancing the lubricants and liquids coolant in the systems of coolingair radiator. That is why a wide range of methods are used for the heat transmission problems solving. In 2016 A. Hussanan et al. [12] have solved the particular heat transfer problem exactly.

The hydrodynamic stability of the Couette flow of an electrically conducting fluid flowing through a porous medium in a parallel-plate channel with a normal magnetic field is investigated in the article [13]. Our results are devoted mostly to distribution of instable area in space unlike the results of the paper [13] where speed, viscosity and magnetic field influence on the flow stability have been explored.

The present paper concentrates mostly on the case of water treatment especially in industry applications. Because of this reason we mostly deal with concentrations instead of heat. But we, of course, can use the similar methods. The enhancement of heat transmission basically depends on the thermal conductivities of nanoparticles, the volume concentration of particles and the mass flow rate. Almost the same parameters are under consideration in the case of the present paper.

\section{Materials and Methods}

The schematic outlook of the channel is shown in the Figure 1. A semi-infinite rectangular channel is considered as a model of a narrow channel inside a sponge, and it is supposed that the channel is symmetric, and the solution can be reflected to the other halfplane. The walls of the channel are partially penetrable, and they are shifting with constant velocity $\dot{a}=\Omega$. The shift may be both stressing $(\Omega<0)$ and stretching $(\Omega>0)$. In the experiment the walls squeezed at a uniform rate $\Omega<0$, and porosity value is $V \in(0,1)$. The fluid of the flow is viscid nanofluid of $(\mathrm{Fe} 3 \mathrm{O} 4 / \mathrm{H} 2 \mathrm{O})$. In the flow there is contaminant which has known mass and concentration values in standard laboratory IUPAC conditions.

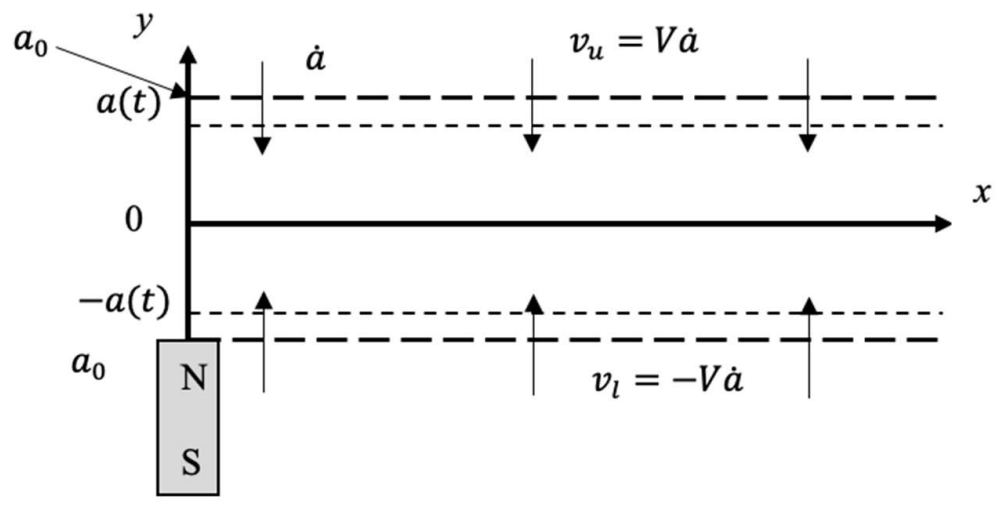

Figure 1. Schematic vision of the channel and flow. 
The additional assumptions in the model are the next:

- Before the flow starts moving, there is homogeneous fluid inside the channel and nanoparticles are not aggregated and not adsorbed; this can be physically made by sonicator;

- The start of the considered process coincides with the moment when the sonicator turns off, the squeezing starts, and the magnetic field is applied;

- It is supposed that the further mentioned values had been gained in other experiments and they are known:

$\circ$ any nanoparticle $(\mathrm{N})$ has the initial aggregation ability $0 \leq A_{a 0} \leq 1$ value, that shows what part of the aggregable particles will be really aggregated, and the initial adsorption ability $0 \leq A_{s 0} \leq 1$ value, that shows what part of the adsorbable particles will be really adsorbed; the values are functions on time and position in general, but they do not depend on position at the initial time;

- adsorption of the nanoparticles $(\mathrm{N})$ means that a particle of contaminant (C) being inside the $r_{s}$-ball around a fixed nanoparticle, will be attached to the nanoparticle during the time $\tau_{s}$, and the mass of the nanoparticle will be increased by the mass of the attached particle; in the same time the aggregation ability $A_{a}$ decreases by $k_{s a}$ times, adsorption ability $A_{s}$ decreases by $k_{s s}$ times;

- aggregation of the nanoparticles $(\mathrm{N})$ means that a nanoparticle being inside the $r_{a}$-ball around a fixed nanoparticle, will be attached to the nanoparticle during the time $\tau_{a}$, and the fixed nanoparticle mass will be increased by the mass of the attached particle; in the same time the aggregation ability $A_{a}$ decreases by $k_{a a}$ times, adsorption ability $A_{s}$ decreases by $k_{\text {as }}$ times.

Let the fluid has volume density at the point $(x, y)$ equal to

$$
\rho=m_{w} n_{w}+m_{c} n_{c}+m_{v} n_{v}
$$

where $m$ is particle's mass, $n$ is concentration in the fluid and the indices denote water $(\mathrm{w})$, contaminant (c) and nanoparticles $(v)$. We denote as $\delta_{c}=\frac{\bar{n}_{w}}{\bar{n}_{c}}, \delta_{v}=\frac{\bar{n}_{w}}{\bar{n}_{v}}$ the concentration factors for contaminant and nanoparticles, where overlined values are related to the standard laboratory conditions.

In order to make the expressions more readable, we use the following notations for the constants and functions. New constants are defined as follows

$$
\begin{gathered}
\alpha_{s}=\frac{4 \pi r_{s}^{3}}{3 \tau_{s}}, \alpha_{a}=\frac{4 \pi r_{a}^{3}}{3 \tau_{a}}, \\
c_{s s}=\frac{1-k_{s s}}{\tau_{s} k_{s s}}, c_{a s}=\frac{1}{k_{a s} \tau_{s}}, c_{a a}=\frac{1-k_{a a}}{\tau_{a} k_{a a}}, c_{s a}=\frac{1}{k_{s a} \tau_{a}},
\end{gathered}
$$

and new functions will be the next

$$
\mathrm{A}_{s}=\frac{A_{s}}{n_{c}}, \mathrm{~A}_{a}=\frac{A_{a}}{n_{v}} .
$$

Abovementioned propositions concerning the model imply the following differential equations

$$
\begin{gathered}
\frac{d m_{v}}{d t}=\partial_{t} m_{v}+u \partial_{x} m_{v}+v \partial_{y} m_{v}=\alpha_{s} m_{c} n_{c}^{2} \mathrm{~A}_{s}+\alpha_{a} n_{v}^{2} m_{v} \mathrm{~A}_{a} \\
\frac{d n_{c}}{d t}=\partial_{t} n_{c}+u \partial_{x} n_{c}+v \partial_{y} n_{c}=-\alpha_{s} n_{c}^{2} n_{v} \mathrm{~A}_{s}
\end{gathered}
$$




$$
\begin{gathered}
\frac{d n_{v}}{d t}=\partial_{t} n_{v}+u \partial_{x} n_{v}+v \partial_{y} n_{v}=-\alpha a n_{v}^{3} \mathrm{~A}_{a}, \\
\frac{d \mathrm{~A}_{s}}{d t}=\partial_{t} \mathrm{~A}_{s}+u \partial_{x} \mathrm{~A}_{s}+v \partial_{y} \mathrm{~A}_{s}=c_{s s} \mathrm{~A}_{s}+c_{a s} \mathrm{~A}_{a}, \\
\frac{d \mathrm{~A}_{a}}{d t}=\partial_{t} \mathrm{~A}_{a}+u \partial_{x} \mathrm{~A}_{a}+v \partial_{y} \mathrm{~A}_{a}=c_{a a} \mathrm{~A}_{a}+c_{s a} \mathrm{~A}_{s} .
\end{gathered}
$$

We have continuity and Navier-Stokes equations additionally:

$$
\begin{gathered}
\dot{\rho}+\rho \partial_{x} u+\rho \partial_{y} v+u \partial_{x} \rho+v \partial_{y} \rho=0, \\
\begin{array}{r}
\rho\left(\partial_{t} u+u \partial_{x} u+v \partial_{y} u\right) \\
=-\partial_{x} p+\eta\left(\partial_{x x} u+\partial_{y y} u\right)-\left(\zeta+\frac{\eta}{3}\right)\left(\partial_{x x} u+\partial_{x y} v\right)+n_{v} \hat{f}_{x},
\end{array} \\
\begin{array}{r}
\rho\left(\partial_{t} v+u \partial_{x} v+v \partial_{y} v\right) \\
=-\partial_{y} p+\eta\left(\partial_{x x} v+\partial_{y y} v\right)-\left(\zeta+\frac{\eta}{3}\right)\left(\partial_{x y} u+\partial_{y y} v\right)+n_{v} \hat{f}_{y},
\end{array}
\end{gathered}
$$

where

$$
\rho=\rho_{w}+\left(m_{c}-m_{w} \delta_{c}\right) n_{c}+\left(m_{v}-m_{w} \delta_{v}\right) n_{v} .
$$

The auxiliary conditions are as follow

$$
\begin{gathered}
u=0, v=-V \Omega \text { for } y=-a, \forall t \neq 0, \forall x, \\
u=0, v=V \Omega \text { for } y=a, \forall t \neq 0, \forall x, \\
\partial u=\partial v=0 \text { for } y= \pm a, \forall t \neq 0, \forall x
\end{gathered}
$$

and there are initial values of the variables at the start of the process:

$$
\begin{gathered}
n_{v 0}=n_{v_{\mid t=0}}, n_{c 0}=n_{c \mid t=0^{\prime}}, m_{v 0}=m_{v_{\mid t=0^{\prime}}} \\
A_{a 0}=A_{a \mid t=0^{\prime}}, A_{s 0}=A_{s \mid t=0^{\prime}}
\end{gathered}
$$

where we have no dependance on $x, y$ for this paper consideration.

Two equations (11) and (12) can be formulated as one equation with $p$ function eliminated. Therefore, we have seven equations totally: Eqs. (5)-(10) plus one Eq. made from (11) and (12). There are seven dependent variables $\left(u, v, n_{v}, n_{c}, m_{v}, \mathrm{~A}_{a}, \mathrm{~A}_{s}\right)$ and three independent variables $(x, y, t)$. The system has total order equal to 9 and we have relevant number of the boundary conditions because the auxiliary conditions operate with values which are uniform in space.

According to the Figure 1 and the model assumptions, the magnetic and gravity fields components which act on the magnetic momentum $m$ and mass $m_{v}$, have the next form

$$
\begin{gathered}
\hat{f}_{x}=3 H \mathfrak{m} \frac{-6 x^{3}(y+a)^{2}-x^{5}-5 x(y+a)^{4}}{\left(x^{2}+(y+a)^{2}\right)^{\frac{7}{2}} \sqrt{\left(x^{2}+2(y+a)^{2}\right)^{2}+x^{2}(y+a)^{2}}} \\
\hat{f}_{y}=-m_{\nu} g-12 H \mathfrak{m} \frac{(y+a)^{3}}{\left(x^{2}+(y+a)^{2}\right)^{\frac{5}{2}} \sqrt{\left(x^{2}+2(y+a)^{2}\right)^{2}+x^{2}(y+a)^{2}}}
\end{gathered}
$$

where $H$ is relevant constant, and it is easy to see that

$$
\partial_{y} \hat{f}_{x}-\partial_{x} \hat{f}_{y}=0
$$


It is clear, that constant homogeneous magnetic field does not force the nanoparticles to move. That is why we need in non-homogeneous magnetic field to attract nanoparticles to the bottom of the channel. The corresponding vector field configurations for the initial point and another three positions when squeezing implied are shown on the Figure 2, where it should be noted that the lengths of arrows are not in the same scale for all shots. Actually, at the last stage the magnetic field is the strongest, but at the same time its heterogeneity is the highest at the moment and it acts farther along $x$ axis.

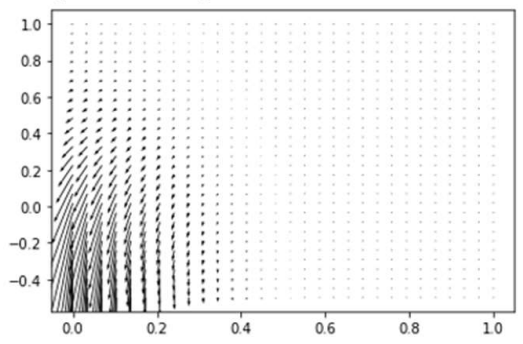

(a)

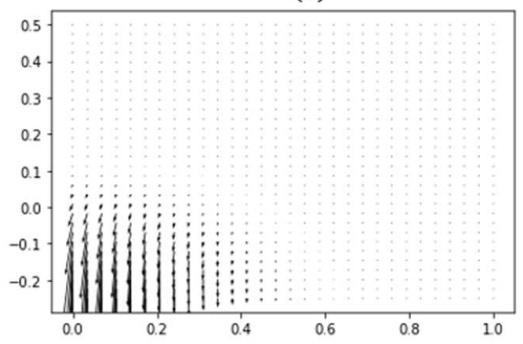

(c)

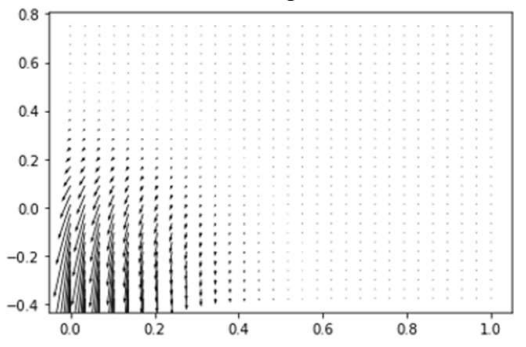

(b)

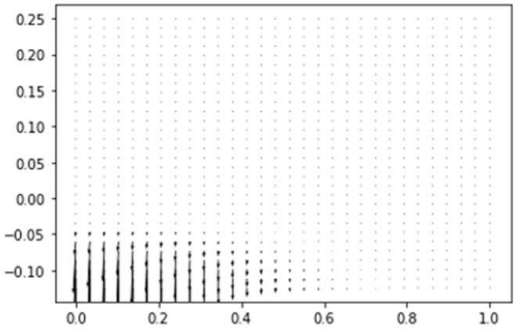

(d)

Figure 2. Magnetic field configurations for start squeezing (a), $1 / 4$ squeezing stroke (b), 1/2 squeezing stroke (c) and $3 / 4$ squeezing stroke $(d)$.

\section{Results}

\subsection{The problem solving}

First of all, we should note that the only time factor in the system is walls moving rate $\Omega$. Hence it is natural to unify the time with $y$ coordinate using new independent variable

$$
\xi=\frac{y}{a}, a=\Omega t+a_{0}
$$

which is unitless and runs over $[-1,1]$. The unification can be made while the rate $\Omega$ is constant in the model and we can write

$$
\partial_{y}=\frac{1}{a} \partial_{\xi}, \partial_{t}=-\frac{\xi \Omega}{a} \partial_{\xi}
$$

Let us try to eliminate the second variable in order to transform the problem to the dynamical system formulation. If we put

$$
\partial_{t}+u \partial_{x}+v \partial_{y} \equiv \partial_{z}
$$

for some (in general, complex) variable $z$, then we will have

$$
u \partial_{x}+\frac{v-\xi \Omega}{a} \partial_{\xi}=\partial_{z}, \partial_{\xi}=\frac{a}{v-\xi \Omega}\left(\partial_{z}-u \partial_{x}\right)
$$

and it follows from (5) that:

$$
\partial_{z} m_{v}=\alpha_{s} m_{c} n_{c}^{2} \mathrm{~A}_{s}+\alpha_{a} n_{v}^{2} m_{v} \mathrm{~A}_{a}
$$


and so on. We have the next transformation rules

$$
\begin{gathered}
(x, \xi) \leftrightarrow(x, z) \\
\partial_{x} x=1, \quad \partial_{x} \xi=0 \\
\partial_{z} x=u, \quad \partial_{z} \xi=\frac{v-\xi \Omega}{a} \\
\partial_{x} x=1, \quad \partial_{x} z=0 \\
\partial_{\xi} x=-\frac{a u}{v-\xi \Omega}, \quad \partial_{\xi} z=\frac{a}{v-\xi \Omega}
\end{gathered}
$$

In order to gain ODE system, we should find expressions in terms of $z, x$ variables for all derivatives involved in (11), (12), and it is necessary to make identity from the Eq. (10).

First of all, let us note that in coordinates $x, \xi$ we obviously have the next:

$$
\begin{array}{ll}
\partial_{x} u=0, & \partial_{\xi} u=\frac{a}{v-\xi \Omega} \partial_{z} u, \\
\partial_{x} v=0, & \partial_{\xi} v=\frac{a}{v-\xi \Omega} \partial_{z} v,
\end{array}
$$

and it is possible to evaluate $v$ explicitly from the Eq. (10) that transforms to the following one:

$$
-\frac{\partial_{z} v}{v-\xi \Omega}=\frac{\partial_{z} \rho}{\rho} .
$$

From here we have

$$
\frac{\rho_{0} v_{0}}{\rho} e^{-\frac{\Omega}{a} z}+\xi \Omega=v
$$

with some $\rho_{0}(x) v_{0}$. Taking $z$ from here and substituting it into (25), we find

$$
\begin{gathered}
z=\frac{a}{\Omega} \ln \frac{\rho_{0} v_{0}}{\rho(v-\xi \Omega)} \\
\partial_{\xi} Z=\frac{a}{v-\xi \Omega}=\frac{a}{\Omega} \rho(v-\xi \Omega)\left(-\frac{\partial_{\xi} \rho}{\rho^{2}(v-\xi \Omega)}-\frac{\left(\partial_{\xi} v-\Omega\right)}{\rho(v-\xi \Omega)^{2}}\right) \\
1=\left(-\frac{\partial_{\xi} \rho(v-\xi \Omega)}{\Omega \rho}-\frac{\partial_{\xi} v}{\Omega}+1\right), \\
\frac{\partial_{\xi} \rho}{\rho}=-\frac{\partial_{\xi} v}{v-\xi \Omega} .
\end{gathered}
$$

Eq. (32) combined with (27) shows that $\frac{\rho_{0} v_{0}}{\rho}$ doesn't depend on $x$. Integration of the Eq. (27) gives the solution for $v$ :

$$
v=\xi \Omega+(1-V) \Omega e^{i \frac{\pi(1+\xi)}{2}},
$$

that obviously obeys the conditions on $v$ in real part. From here

$$
\frac{\rho_{0} v_{0}}{\rho} \equiv R(z)=(1-V) \Omega e^{\frac{\Omega}{a} z+i \frac{\pi(\xi+1)}{2}} .
$$

Let us now solve Eqs. (5)-(9) which are equations on the only independent variable $z$ :

$$
\begin{gathered}
\partial_{z} m_{v}=\alpha_{s} m_{c} n_{c}^{2} \mathrm{~A}_{s}+\alpha_{a} n_{v}^{2} m_{v} \mathrm{~A}_{a} \\
\partial_{z} n_{c}=-\alpha_{s} n_{c}^{2} n_{v} \mathrm{~A}_{s} \\
\partial_{z} n_{v}=-\alpha_{a} n_{v}^{3} \mathrm{~A}_{a}
\end{gathered}
$$




$$
\begin{aligned}
& \partial_{z} \mathrm{~A}_{s}=c_{s s} \mathrm{~A}_{s}+c_{a s} \mathrm{~A}_{a}, \\
& \partial_{z} \mathrm{~A}_{a}=c_{a a} \mathrm{~A}_{a}+c_{s a} \mathrm{~A}_{s} .
\end{aligned}
$$

The solution can be found easily, and it has the following form:

$$
\begin{gathered}
n_{v}(z)=\left(2 \alpha_{a} \int_{0}^{z} \mathrm{~A}_{a}(\zeta) d \zeta+\frac{1}{n_{v 0}^{2}}\right)^{-\frac{1}{2}}, \\
n_{c}(z)=\frac{1}{\alpha_{s}}\left(\frac{1}{\alpha_{s} n_{c 0}}+\int_{0}^{z} \mathrm{~A}_{s}(\zeta) n_{v}(\zeta) d \zeta\right)^{-1}, \\
m_{v}(z)=-m_{c} \frac{n_{c}(z)-n_{c 0}}{n_{v}(z)}+\frac{n_{v 0} m_{v 0}}{n_{v}(z)}, \\
\mathrm{A}_{s}=F_{1} e^{b_{1} z}+F_{2} e^{b_{2} z} \\
\mathrm{~A}_{a}=F_{1} \frac{b_{1}-c_{s s}}{c_{a s}} e^{b_{1} z}+F_{2} \frac{b_{2}-c_{s s}}{c_{a s}} e^{b_{2} z},
\end{gathered}
$$

where

$$
b_{1,2}=\frac{c_{a a}+c_{s s} \pm \sqrt{\left(c_{a a}+c_{s s}\right)^{2}-4\left(c_{a a} c_{s s}-c_{a s} c_{s a}\right)}}{2},
$$

and, in general,

$$
F_{1}=\frac{\mathrm{A}_{a 0} c_{a s}-\mathrm{A}_{s 0}\left(b_{2}-c_{s s}\right)}{\sqrt{\left(c_{a a}+c_{s s}\right)^{2}-4\left(c_{a a} c_{s s}-c_{a s} c_{s a}\right)}}, \quad F_{2}=\frac{-\mathrm{A}_{a 0} c_{a s}+\mathrm{A}_{s 0}\left(b_{1}-c_{s s}\right)}{\sqrt{\left(c_{a a}+c_{s s}\right)^{2}-4\left(c_{a a} c_{s s}-c_{a s} c_{s a}\right)}},
$$

with index 0 corresponding to $z=0$ position.

In consequence of (11), (12), by equivalence of mixed derivatives, we derive:

$$
\begin{aligned}
-\partial_{y} \rho\left(\partial_{t} u+u \partial_{x} u\right. & \left.+v \partial_{y} u\right) \\
& -\rho\left(\partial_{t y} u+\partial_{y} u \partial_{x} u+\partial_{y} v \partial_{y} u+u \partial_{x y} u+v \partial_{y y} u-\partial_{t x} v-\partial_{x} u \partial_{x} v\right. \\
& \left.-\partial_{x} v \partial_{y} v-u \partial_{x x} v-v \partial_{y x} v\right)+\eta\left(\partial_{x x y} u+\partial_{y y y} u-\partial_{x x x} v-\partial_{x y y} v\right) \\
& -\left(\zeta+\frac{\eta}{3}\right)\left(\partial_{x x y} u+\partial_{x y y} v-\partial_{x x y} u-\partial_{x y y} v\right) \\
& +\partial_{x} \rho\left(\partial_{t} v+u \partial_{x} v+v \partial_{y} v\right)+\hat{f}_{x} \partial_{y} n_{v}-\hat{f}_{y} \partial_{x} n_{v}=0 .
\end{aligned}
$$

This complicated equation can be essentially simplified using our previous considerations. Thus, involving Eqs. (25), (26) and (27), we have from (47)

$$
\begin{aligned}
-\left(\frac{\partial_{z} \rho}{\rho}+\frac{\Omega}{a}\right) \partial_{z} u & -\partial_{z z} u \\
& +\frac{\eta \rho}{\rho_{0}^{2} v_{0}^{2}} e^{2 \frac{\Omega}{a} z}\left(\left(4 \frac{\partial_{z} \rho}{\rho} \frac{\Omega}{a}+2 \frac{\Omega^{2}}{a^{2}}+\frac{\partial_{z z} \rho}{\rho}+\frac{\left(\partial_{z} \rho\right)^{2}}{\rho^{2}}\right) \partial_{z} u\right. \\
& \left.+3\left(\frac{\partial_{z} \rho}{\rho}+\frac{\Omega}{a}\right) \partial_{z z} u+\partial_{z z z} u\right)+\frac{\hat{f}_{x}}{\rho} \partial_{z} n_{v}-\left(\frac{\hat{f}_{y}}{\rho_{0}} v_{0} e^{\frac{\Omega}{a}}+\frac{\hat{f}_{x}}{\rho} u\right) \partial_{x} n_{v}=0,
\end{aligned}
$$

Let us look for solutions in the form of

$$
u^{\prime}=U R e^{-\frac{\Omega}{a} z}
$$

for some constant $U$ and note that 


$$
\partial_{x} n_{v}=0
$$

Then from (48) we derive

$$
\begin{gathered}
-\left(-\frac{i \pi R}{2 a} e^{-\frac{\Omega}{a} z}\right) u^{\prime}-u^{\prime \prime}+\frac{\eta \rho}{\rho_{0}^{2}} e^{2 \frac{\Omega}{a} z}\left(\left(-\frac{\pi^{2}}{4 a^{2}} R^{2} e^{-2 \frac{\Omega}{a} z}\right) u^{\prime}+3\left(-\frac{i \pi R}{2 a} e^{-\frac{\Omega}{a} z}\right) u^{\prime \prime}+u^{\prime \prime \prime}\right) \\
+\frac{\hat{f}_{x}}{\rho} \partial_{z} n_{v}=0 .
\end{gathered}
$$

While we have from (34)

$$
R^{\prime}=R \frac{\Omega}{a}+\frac{i \pi}{2 a} R^{2} e^{-\frac{\Omega}{a} z}, \quad R^{\prime \prime}=R \frac{\Omega^{2}}{a^{2}}+\frac{i \pi}{a} \frac{\Omega}{a} R^{2} e^{-\frac{\Omega}{a} z}-\frac{\pi^{2}}{2 a^{2}} R^{3} e^{-2 \frac{\Omega}{a} z},
$$

then

$$
\begin{gathered}
u^{\prime \prime}=U \frac{i \pi}{2 a} R^{2} e^{-2 \frac{\Omega}{a} z}, \\
u^{\prime \prime \prime}=-U \frac{\pi^{2}}{2 a^{2}} R^{3} e^{-3 \frac{\Omega}{a} z}, \\
\partial_{z} \ln R=\frac{\Omega}{a}+\frac{i \pi}{2 a} R e^{-\frac{\Omega}{a} z}, \\
\partial_{z z} \ln R=-\frac{\pi^{2}}{4 a^{2}} R^{2} e^{-2 \frac{\Omega}{a} z} .
\end{gathered}
$$

Substitution into the Eq. (51) shows that the function (49) turns to zero all terms in (51) except the last one. In order to solve the equation, let us use the variation of the constant method. Suppose that $U=U(z)$. Then

$$
\begin{gathered}
u^{\prime \prime}=U \frac{i \pi}{2 a} R^{2} e^{-2 \frac{\Omega}{a} z}+U^{\prime} R e^{-\frac{\Omega}{a} z}, \\
u^{\prime \prime \prime}=-U \frac{\pi^{2}}{2 a^{2}} R^{3} e^{-3 \frac{\Omega}{a} z}+U^{\prime} \frac{i \pi}{a} R^{2} e^{-2 \frac{\Omega}{a} z}+U^{\prime \prime} R e^{-\frac{\Omega}{a} z},
\end{gathered}
$$

and we have from (51) that

$$
U^{\prime}\left(\rho_{0} v_{0} e^{-\frac{\Omega}{a} z}+\frac{i \pi \eta}{2 a}\right)-\frac{U^{\prime \prime} \eta}{R} e^{\frac{\Omega}{a} z}-\hat{f}_{x} \partial_{z} n_{v}=0,
$$

This equation can help us to solve the first problem: to find a flow stability conditions.

\subsection{Stability of the flow}

In order to find the stability conditions, we need to formulate the very definition of the stability. The stability brakes when velocities have chance to become infinite and the critical condition is

$$
u=\sum_{i} \hat{u}_{i} e^{\omega_{c i} t}, \quad \max _{i}\left(\operatorname{Re} \omega_{c i}\right)=0,
$$

where $\hat{u}_{i}$ is allowed to be at most polynomial in time. The flow is stable when all $\operatorname{Re} \omega_{i}<$ 0 and it is unstable when for at least one $i$ holds $\operatorname{Re} \omega_{i}>0$. Of course, the same is actual for other velocity component $v$. Let us recall the first equation of (52) and solve it

$$
R=e^{\frac{\Omega}{a} z}\left(\frac{1}{v_{0}}-\frac{i \pi}{2 a} z\right)^{-1}
$$

where $v_{0}=R(0)$. It is easy to derive that

$$
v_{0}=i(1-V) \Omega e^{i \frac{\pi \xi_{0}}{2}}, \quad z=\frac{2 a}{\pi(1-V) \Omega}\left(e^{-i \frac{\pi \xi}{2}}-e^{-i \frac{\pi \xi_{0}}{2}}\right),
$$

where $\xi_{0}$ is value of $\xi$ that corresponds to $z=0$. 
Let us consider the vertical velocity $v$. Taking into account that $z$ is time equivalent, we have the following stability condition from (28) and (60):

$$
-R e\left(\frac{\Omega}{a} z\right)<0 \text {. }
$$

Recalling that $\Omega<0$ when squeezing and $\Omega>0$ when stretching, we have from (62) and (63) that the flow is stable when:

$$
\text { squeezing: } \cos \frac{\pi \xi}{2}<\cos \frac{\pi \xi_{0}}{2}, \text { stratching: } \cos \frac{\pi \xi}{2}>\cos \frac{\pi \xi_{0}}{2}
$$

In particular, if there is no magnetic field applied, just $\xi_{0}=0$ has physical sense and hence we have very simple conditions

- The flow has no stability restrictions from $v$ component when squeezing;

- The flow is always unstable when stretching.

However, in the presence of the magnetic field, the value of $\xi_{0}$ may be non-zero and we will have something like the following (the restrictions derived just from $v$ component)

- The flow is stable for $|\xi|>\left|\xi_{0}\right|$ and unstable for $|\xi|<\left|\xi_{0}\right|$ when squeezing;

- The flow is stable for $|\xi|<\left|\xi_{0}\right|$ and unstable for $|\xi|>\left|\xi_{0}\right|$ when stretching.

Now let us turn to $u$ velocity component and consider the flow under magnetic field influence. From (34), (40), (49) and (59) we can find that all possible exponents in the expression of $u$ are the next:

$$
\left(B-\frac{\Omega}{a}\right) z, B z,\left(B+\frac{\Omega}{a}\right) z,-\frac{1}{2} b_{1} z,\left(b_{1}-\frac{3}{2} b_{2}\right) z,\left(b_{2}-\frac{3}{2} b_{1}\right) z,-\frac{1}{2} b_{2} z,
$$

where $B$ is an exponential factor of $\frac{u}{\hat{f}_{x}}$ in "time" $z$. All values in (65) must have negative real parts to provide flow stability. It follows from here and from conditions for $v$, that for squeezing the conditions take the form

$$
z_{r}<0, i B z_{i}<-\frac{\Omega}{a} z_{r}, 0>\frac{2}{3} b_{2}>b_{1}>\frac{3}{2} b_{2} .
$$

In case of stretching $B=\max _{i} B_{i}$ and inequalities are the following

$$
z_{r}>0, i B z_{i}<-\frac{\Omega}{a} z_{r}, 0<\frac{2}{3} b_{2}<b_{1}<\frac{3}{2} b_{2} .
$$

Using (18), we can approximate the value of $B$ as the next

$$
B=i \frac{5(1-V) \Omega}{a\left(\xi_{0}+1\right)} .
$$

Thus, the MHD-flow is stable when the following conditions hold

- $\quad$ Squeezing $(\Omega<0):|\xi|>\left|\xi_{0}\right|, 0>\frac{2}{3} b_{2}>b_{1}>\frac{3}{2} b_{2}$;

- $\quad$ Stretching $(\Omega>0):|\xi|<\left|\xi_{0}\right|, 0<\frac{2}{3} b_{2}<b_{1}<\frac{3}{2} b_{2}$ with

$$
\frac{5(1-V)}{\xi_{0}+1}<\frac{\cos \frac{\pi \xi}{2}-\cos \frac{\pi \xi_{0}}{2}}{\sin \frac{\pi \xi_{0}}{2}-\sin \frac{\pi \xi}{2}}
$$

as common condition.

\section{Discussion}

In order to illustrate our model, the colloid fluid based on water with calcium hydro carbonate of $20 \%$ (that is very hard water) is considered and $\mathrm{Fe}_{3} \mathrm{O}_{4}$ nanoparticles were used. The following parameters correspond to the chosen fluid:

$$
\begin{aligned}
r_{s} & =2.1013 * 10^{-8}, r_{a}=1.41842 * 10^{-8}, \\
\tau_{s} & =4.2026 * 10^{-2}, \tau_{a}=2.83684 * 10^{-2}, \\
k_{a a} & =1.1, k_{a s}=10.2, k_{s a}=10.5, k_{s s}=1.6,
\end{aligned}
$$


all values except $k .$. have units from SI. The values of parameters are evaluated from reference values [14].

We have built graphs for $n_{v}(\xi)$ for fixed $a_{0}=1 \mathrm{sm}$, and $\Omega=-0.005 \mathrm{~m} / \mathrm{s}$ under magnetic field made by permanent alnico magnet that gives induction about $0.6 \mathrm{~T}$ and $0.9 \mathrm{~T}$ in the form as shown on the Figure 2.

It is possible to determine the distribution of nanoparticles concentration using the Eqs. (40) - (44) and definition of $z(\xi)$ from (62). The formula is too heavy, and we illustrate it by graph.

On the Figure 3 the situation without magnetic field is shown for the cases of standard nanoparticles concentration (green color), 20\% reduced nanoparticles concentration (blue color) and $20 \%$ increased nanoparticles concentration (red color). It is clear that concentration oscillates inside unstable zone and takes constant value in stable zone. In the same time, it is notable, that amplitude of the oscillations grows together with nanoparticles concentration: in particular, the relative concentration decreases outside the turbulent zone. However, the form of the oscillations does not change.

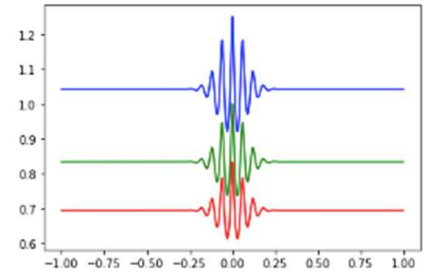

(a)

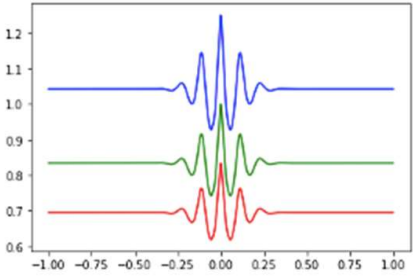

(b)

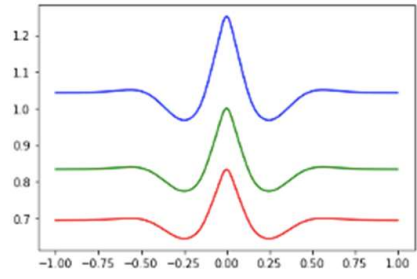

(c)

Figure 3. Relative concentration of nanoparticles versus vertical position without magnetic field, at $\mathrm{t}=1 \mathrm{sec}(\mathrm{a}), 10 \mathrm{sec}$ (b) and $18 \mathrm{sec}(\mathrm{c})$ : reduced conc. (blue), standard conc. (green), increased conc. (red).

The result for magnetic field of $0.6 \mathrm{~T}$ impact is shown on the Figure 4, and Figure 5 shows case of $0.9 \mathrm{~T}$. It can be noted that magnetic field shifts down the maximum of the (c) curve. Another one notable thing is that concentration decreases almost to zero inside the turbulent zone under strong enough magnetic field. This result is new.

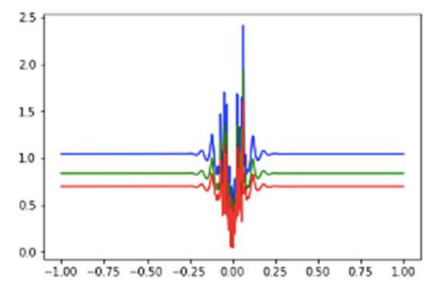

(a)

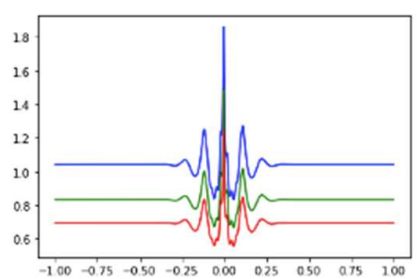

(b)

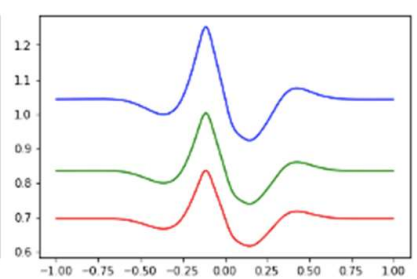

(c)

Figure 4. Relative concentration of nanoparticles versus vertical position under 0.6 T magnetic field, at $t=1 \mathrm{sec}(\mathrm{a}), 10 \mathrm{sec}$ (b) and $18 \mathrm{sec}$ (c): reduced conc. (blue), standard conc. (green), increased conc. (red).

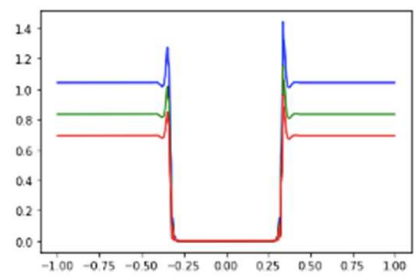

(a)

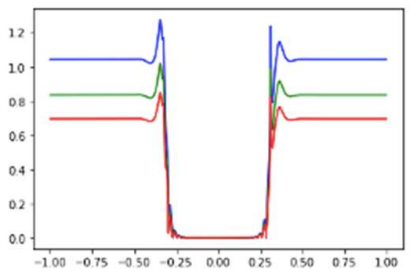

(b)

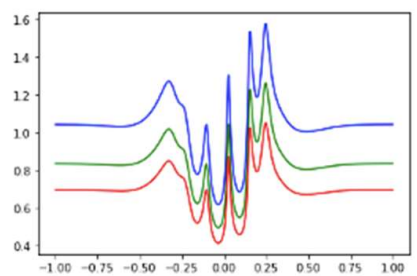

(c) 
Figure 5. Relative concentration of nanoparticles versus vertical position under $0.9 \mathrm{~T}$ magnetic field, at $t=1 \mathrm{sec}$ (a), $10 \mathrm{sec}$ (b) and $18 \mathrm{sec}$ (c): reduced conc. (blue), standard conc. (green), increased conc. (red).

It should be noted that all presented curves were constructed by numeric evaluation, and they are just approximations. However, the underlined effects are confirmed for any large number of steps.

As it is clear from the Figures 3 - 5, the presence of magnetic field does not affect concentration of nanoparticles in the laminar layers of the flow. However, it has extremely high influence on the concentration inside the turbulent layer: it decreases almost to zero on the squeezing stroke whenever the magnetic field is strong enough. It is possible to show the influence of the magnetic field on the nanoparticles concentration inside the turbulent layer by average concentration versus field magnitude graph, shown on the Figure 6.

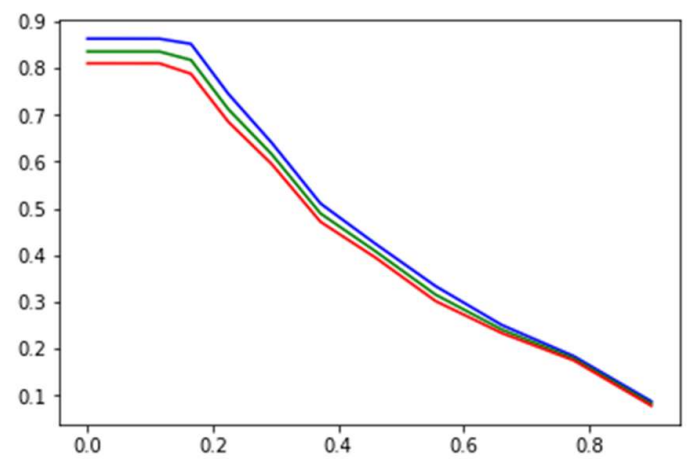

Figure 6. Relative concentration of nanoparticles versus magnetic field magnitude at $t=1 \mathrm{sec}$ : reduced conc. (blue), standard conc. (green), increased conc. (red).

It may be helpful to see what happen with the nanoparticles concentration when the squeezing rate changes. Let us show the concentrations versus vertical position for the cases of the rate decreases and increases twice. Please consult Figure 7 and Figure 8, where just standard concentrations are considered.

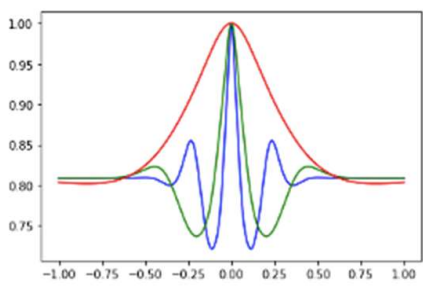

(a)

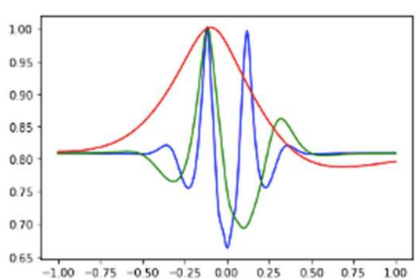

(b)

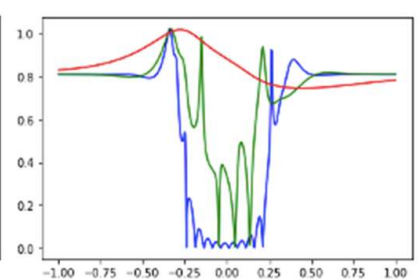

(c)

Figure 7. Relative concentration of nanoparticles versus vertical position under $0 \mathrm{~T}(\mathrm{a}), 0.6 \mathrm{~T}(\mathrm{~b})$ and $0.9 \mathrm{~T}$ (c) magnetic field with double squeezing rate, at $\mathrm{t}=0.5 \mathrm{sec}$ (blue), $5 \mathrm{sec}$ (green) and $9 \mathrm{sec}$ (red). 


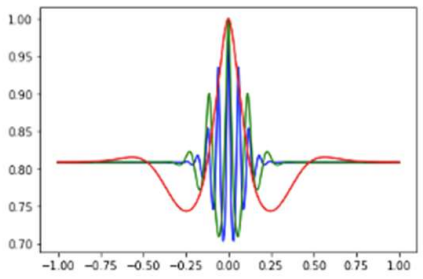

(a)

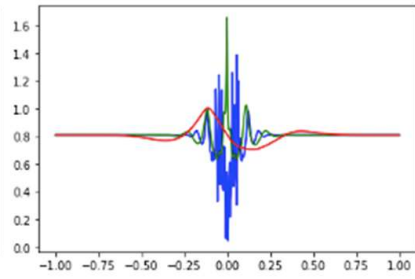

(b)

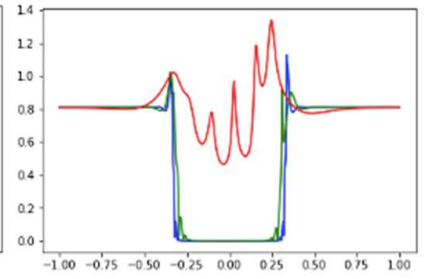

(c)

Figure 8. Relative concentration of nanoparticles versus vertical position under $0 \mathrm{~T}(\mathrm{a}), 0.6 \mathrm{~T}(\mathrm{~b})$ and $0.9 \mathrm{~T}$ (c) magnetic field with half squeezing rate, at $\mathrm{t}=2 \mathrm{sec}$ (blue), $20 \mathrm{sec}$ (green) and $36 \mathrm{sec}$ (red).

The graphs show that low squeezing rate brings better result: clearness in turbulent layer stays for increased duration. However, it is clear that this dependency is nonlinear and the best rate should be determined specially.

\section{Conclusions}

The following highlights are related to this work:

1. It was new concept proposed to consider the flows of Poiseuille and Couette with multicomponent liquids: using the complex independent variable (with time-like real part) that considers the inner time of the flow;

2. Stability conditions of the nano-liquid flow was formulated both for squeezing and for stretching of the flow;

3. It was shown that there are oscillations of nanoparticles concentration across the channel when the magnetic field is absent. These oscillations attenuate with time;

4. It was shown that nanoparticles concentration becomes irregularly oscillated under weak magnetic field. The concentration in turbulent part of flow becomes almost zero in strong magnetic field.

Author Contributions: Conceptualization, Z.Hussain and S.Zuev; methodology, S.Zuev; software, S.Zuev; validation, Z.Hussain, S.Zuev. and P.Kabalyants; formal analysis, S.Zuev; investigation, S.Zuev; resources, Z.Hussain; data curation, P.Kabalyants; writing-original draft preparation, S.Zuev.; writing - review and editing, Z.Hussain; visualization, S.Zuev; supervision, S.Zuev; project administration, S.Zuev; funding acquisition, S.Zuev and P. Kabalyants. All authors have read and agreed to the published version of the manuscript.

Funding: This research received no external funding.

Conflicts of Interest: The authors declare no conflict of interest.

\section{References}

1. Alfven, H., Existence of electromagnetic-hydrodynamic waves, Nature 1942, Volume 150 (3805), pp. $405-406$.

2. Hussain, Z.; Zeeshan, R.; Shahzad, M. An optimised stability model for the magnetohydrodynamic fluid, Pramana - J. Phys. 2021, 95, p.27.Author 1, A.; Author 2, B. Title of the chapter. In Book Title, 2nd ed.; Editor 1, A., Editor 2, B., Eds.; Publisher: Publisher Location, Country, 2007; Volume 3, pp. 154-196.

3. Hussain, Z.; Ali, M.; Shahzad, M. Optimized wave perturbation for the linear instability of magnetohydrodynamics in plane Poiseuille flow, Pramana - J. Phys. 2020, Volume 94, p. 49.

4. Hussain, Z.; Hussain, S.; Kong, T. Instability of MHD Couette flow of an electrically conducting fluid, AIP Adv. 2018, Volume 8, p. 105209.

5. Hussain, Z.; Zuev, S.; Kabobel, A.; Ali, M.; Sultan, F.; Shahzad, M. MHD instability of two fluids between parallel plates, Appl. Nanosci. 2020, Volume 10, pp. 5211-5218, https://doi.org/10.1007/s13204-020-01361-7.

6. Dalkılıç, A.S.; Yalçın, G.; Küçükyıldırım, B.O.; Öztuna, S.; Eker, A.A.; Jumpholkul, C.;... \& Wongwises, S. Experimental study on the thermal conductivity of water-based CNT-SiO2 hybrid nanofluids. International Communications in Heat and Mass Transfer, 2018, Volume 99, pp. 18-25. 
7. Anisur, R.; Xu, W; Li, K.; Dou, H.-S.; Khoo, B.C.; Mao, J. Influence of Magnetic Force on the Flow Stability in a Rectangular duct. Feb. 2019, Advances in Appl. Math. and Mech., Volume 11(1), pp. 24-37.

8. Hussain, Z.; Abbasi, A.Z.; Ahmad, R.; Bukhari, H.; Shahzad, M.; Sultan, F.; Ali, M. Vibrio cholerae dynamics in drinking water: mathematical and statistical analysis. 2020, Appl. Nanosci., Volume 10, pp. 4519-4522. https://doi.org/10.1007/s13204-020-01292$\underline{3}$

9. Downey, J.P.; Pojman, J.A. Polymer Research in Microgravity: Polymerization and Processing, American Chemical Society: Washington, DC, USA, 2001.

10. Jing, D.; Hu, Y.; Liu, M.; Wei, J.; Guo, L. Preparation of highly dispersed nanofluid and CFD study of its utilization in a concentrating PV/T system. 2015, Sol. Energy, Volume 112, pp. 30-40.

11. Kandelousi, M.S. Effect of spatially variable magnetic field on ferrofluid flow and heat transfer considering constant heat flux boundary condition. 2014, Eur Phys J Plus, Volume 129, pp. 1-2.

12. Hussanan, A.; Khan, I.; Hashim, H.; Mohamed, M.K.A.; Ishak, N.; Sarif, N.M.; Salleh, M.Z. Unsteady MHD flow of some nanofluids past an accelerated vertical plate embedded in a porous medium. 2016, J Teknol, Volume 78, pp. 121-126.

13. Hussain, Z.; Khan, N.; Gul, T.; Ali, M.; Shahzad, M.; Sultan, F. Instability of magneto hydro dynamics Couette flow for electrically conducting fluid through porous media. 2020, Appl. Nanosci., Volume 10, pp. 5125-5134. https://doi.org/10.1007/s13204-020-01307-z

14. Babichev, A.P.; Babushkina, N.A.; Bratkovskii, A.M.;.. Physical values; Energoatomizdat: Moscow, Russia, 1991; pp. 123, 124, 370, 376 [in Russian]. 